\title{
ARTICLE
}

\section{Seismic Structural Response Analysis Considering Fault-Structure System: Application to Nuclear Power Plant Structures}

\author{
Pher Errol B. QUINAY ${ }^{1,2, *}$, Tsuyoshi ICHIMURA ${ }^{1}$, Muneo HORI $^{1}$, M. L. L. WIJERATHNE ${ }^{1}$ and Akemi NISHIDA ${ }^{2}$ \\ ${ }^{1}$ Earthquake Research Institute, University of Tokyo, Yayoi 1-1-1, Bunkyo-ku, Tokyo 113-0032, Japan \\ ${ }^{2}$ Center for Computational Science and e-Systems, Japan Atomic Energy Agency, \\ 5-1-5 Kashiwanoha, Kashiwa, Chiba 277-8587, Japan
}

\begin{abstract}
Accurate estimation of structural responses is important to mitigate loss of life and property. Earthquake ground motions are known to vary spatially and temporally, thereby affecting structural responses. Therefore, we conducted a numerical analysis based on a fault-structure system to account for such variations. We developed a full threedimensional model that includes a fault, irregular crust structure, soft soil basins near the ground surface, and structures at the surface. However, huge computational cost is required to solve these components simultaneously. Ichimura and Hori (2009a) presented a macro-micro analysis method based on singular perturbation expansion to reduce computational cost, but to date, no large-scale application examples have been conducted. We implemented a highly tuned finite-element method code for macro-micro analysis systems to handle large-scale wave propagation in a complicated crust structure as well as the seismic response of a structure at the surface. After verifying the accuracy by comparing the results with analytical Green's function solutions, we demonstrate a seismic response using a simple model of a nuclear power plant structure with actual settings. Our methodology can enable detailed prediction of the seismic response of a nuclear facility, permitting the generation of more reliable estimates of the seismic safety of new or existing nuclear power plant facilities.
\end{abstract}

KEYWORDS: seismic analysis of nuclear power plant structure, fault-structure system, macro-micro analysis, hybrid grid finite element method

\section{Introduction}

Accurate estimation of structural responses during a large earthquake is important to prevent loss of life or significant damage to structures. Large-scale structures situated near faults are very susceptible because ground motions vary spatially and temporally due to phase differences and amplification differences that vary by fault rupture processes and crustsoil structures. These variations can affect the response of a whole structure and its components. A full three-dimensional (3D) analysis can account for these variations, enabling accurate estimation of the seismic response. A 3D model that includes a fault, a 3D irregular crust structure, soft soil basins near the ground surface, and structures at the surface is called a fault-structure system. A direct analysis (i.e., all components of the 3D model are simulated simultaneously) is a straightforward and desirable way to solve the fault-structure system. However, because the target domain size is large $\left(10^{4} \sim 10^{5} \mathrm{~m}\right)$ and the required resolution is fine $\left(10^{-2} \sim 10^{1} \mathrm{~m}\right)$, direct analysis involves a huge computational cost.

To resolve this problem, researchers have proposed alternative methods that permit accuracy comparable to a direct analysis but use fewer computational resources. For example, a two-step hybrid method combining the discrete wavenumber, finite-difference method, and a finite-element method (FEM)

*Corresponding author, E-mail: equinay2@eri.u-tokyo.ac.jp

(C) 2011 Atomic Energy Society of Japan, All Rights Reserved. can simulate P-SV seismic motion and the effects on local topographic and geologic structures. ${ }^{1)}$ Another method involves domain reduction. ${ }^{2)}$ This is a two-step procedure: the model first computes the response in a localized domain by conducting forward modeling (fault to surface) without the local geologic structure; in the second step, it applies the stored results as input boundary conditions at the interface of the second smaller model containing the local structure. Another, more mathematical approach has been proposed; ${ }^{3)}$ this model uses singular perturbation expansion. Application of this expansion to the elastodynamic equation involves a two-step multiscale procedure (macro-micro analysis or MMA) that first computes a solution to the problem in a coarse-resolution model and then adds corrections to the solution to include the effects of a local structure in high resolution. We chose to use MMA because its accuracy has been verified through the modeling of seismic responses of structures and local geologic and soil conditions for small-scale problems. ${ }^{3)}$ Although Reference 3 proposed the MMA, they have not estimated seismic structural responses using a complete fault-structure system setting because to date, no numerical simulation tool has been able to handle large-scale multiscale problems.

In this paper, we present the seismic responses of a simple model of a nuclear power-plant (NPP) structure considering a complete fault-structure system with settings similar to actual 
conditions, using a simulation tool that can handle large-scale problems. The contents of this paper are as follows: (1) a brief description of the MMA; (2) a description of the numerical simulation tool used for the MMA in this study; (3) a numerical verification of the simulation tool; and (4) an application of seismic analysis using a simple model of a NPP during a scenario earthquake using this simulation tool.

\section{Methodology}

MMA can yield results with accuracy comparable to those of direct analysis but involves lower computational cost. ${ }^{3)}$ Figure 1(a) shows the target fault-structure system. This system is decomposed into two subsystems (Fig. 1(b)) that consider the fault-to-surface model in coarse resolution (macro model) and a structure model near the surface in high resolution (micro model). The MMA can rigorously handle this decomposed system. Although the direct analysis must compute all components of the fault-structure system simultaneously, in the MMA we can solve the decomposed system independently using two steps: first, we use the macro model to compute the solution for the system at a geologic-length scale (macro analysis). Next, we use the micro model to refine the solution (by including corrections) for the region surrounding the structure (micro analysis). Because we independently solve the target fault-structure system using two steps, the computation cost is drastically reduced.

The MMA equations are similar to those for conventional linear elastic seismic wave propagation and structural analysis. ${ }^{3)}$ To derive the discretized form of these equations, the Galerkin method can be used for spatial discretization, and the Newmark- $\beta$ method $(\beta=1 / 4)$ can be used for time integration. A Rayleigh damping term can then be added to incorporate material attenuation:

$$
\begin{aligned}
& \left(\mathbf{K}+\frac{2}{\Delta t} \mathbf{C}+\frac{4}{\Delta t^{2}} \mathbf{M}\right) \mathbf{u}^{n+1}= \\
& \left(\frac{2}{\Delta t} \mathbf{C}+\frac{4}{\Delta t^{2}} \mathbf{M}\right) \mathbf{u}^{n}+\left(\mathbf{C}+\frac{4}{\Delta t} \mathbf{M}\right) \mathbf{v}^{n}+\mathbf{M a}^{n}+\mathbf{f}^{n+1},
\end{aligned}
$$

where $\mathbf{K}, \mathbf{C}, \mathbf{M}, \mathbf{u}, \mathbf{v}, \mathbf{a}, \mathbf{f}, \Delta t$, and $n$ are the stiffness matrix, Rayleigh damping matrix, mass matrix, displacement vector, velocity vector, acceleration vector, force vector, time increment, and time step, respectively. The Rayleigh damping term is given by:

$$
\mathbf{C}=\alpha \mathbf{M}+\gamma \mathbf{K},
$$

where $\alpha$ and $\gamma$ are constants computed by solving the following least-squares problem.

$$
\min _{\alpha, \gamma}\left[\int_{\omega_{\min }}^{\omega_{\max }}\left(\frac{1}{2 Q}-\left(\frac{\alpha}{2 \omega}+\frac{\gamma \omega}{2}\right)\right)^{2} d \omega\right]
$$

where $Q$ is the seismic quality factor, and $\omega_{\max }$ and $\omega_{\min }$ are the bounds of minimum and maximum target angular frequency of the problem, respectively.

The accuracy of MMA has been verified for fault-structure systems, but application has been limited to small-scale problems because to date, numerical simulation tools have been unable to handle large-scale problems. ${ }^{3)}$ We computed a faultstructure system with actual settings using the hybrid-grid
FEM, ${ }^{4)}$ which can handle large-scale problems. When solving a fault-structure system, it is important to ensure accuracy at both geologic- and engineering-length scales. For the geologic-length scale model, because crust structure usually has a highly complicated geometry (as evidenced by layer interfaces and topography), our modeling tool is flexible in meshing complicated configurations and satisfying the traction-free boundary conditions. This hybrid-grid FEM utilizes tetrahedral and cubic elements to enable efficient modeling of the local crust structure, surface topography, and structure components with sufficient detail. By incorporating tetrahedral elements for complicated regions, modeling accuracy is improved. The octree technique is also included to handle cubic elements, thereby reducing memory requirements. ${ }^{5)}$ In this technique, because cubic elements are treated as voxels, only a few representative elements must be stored in the memory. ${ }^{6)}$ In seismology, the finite-difference method is used for this kind of numerical simulation, but additional treatment is needed to satisfy the traction-free boundary condition for complicated surface topography. In contrast, the FEM requires no additional treatment because this condition is inherently satisfied in the FEM formulation. In engineering-length scale models, which include local soil structure and structures on the surface, the same technique can be applied with much higher resolution.

\section{Numerical Verification}

We verify the applicability of the simulation tool for computing ground response for a layered structure. We conduct this numerical verification using a horizontally two-layered crust structure with a point source. The first-step analysis solves the macro model, and the second-step analysis solves the micro model. The macro model simulates the seismic response from fault to surface, and the results are then used as a boundary condition for the micro model to simulate the local site response near the surface where a structure may be located.

The details of the macro and micro models are shown in Fig. 2. The problem settings (domain size, material properties, and point-source settings) are given in Tables 1, 2, and $\mathbf{3}$. In both macro and micro models, the target frequency is set at $1.0 \mathrm{~Hz}$, and 10 elements are used for one wavelength. In both macro and micro analyses, the FEM models are generated based on the hybrid-grid method using first-order tetrahedral and tri-linear hexahedral (cube) finite elements, as described in the previous section. The settings result in a maximum element size of $250.0 \mathrm{~m}$ for the softer layer in both macro and micro models. For the transient analysis, the time step is 0.01 second, and the total number of time steps is 4096 steps. Six receivers are placed in the micro model to record $x, y$, and $\mathrm{z}$ displacement; see Fig. 2(c) for the receiver locations. The results are band-pass filtered between $0.1 \mathrm{~Hz}$ and $1.0 \mathrm{~Hz}$.

We compare responses at the receivers computed using the MMA with the Green's function solution as shown in Fig. 3. ${ }^{7)}$ Surface ground motion is reproduced well at all observation points. Quantitative comparison of misfits is also provided in Table 4. Envelope and phase misfits are checked based on misfit criteria, ${ }^{8)}$ which accounts for wavelength time and fre- 
quency information. The obtained envelope and phase misfit values are less than $5 \%$ and $1 \%$, respectively; these results verify the accuracy of the simulation tool for application to fault-structure system analysis.

\section{Application Example}

We conduct a seismic-response analysis of a simplified NPP structure in a hypothetical earthquake scenario. The modeled crust structure is two-layered, with a realistic surface and interface topography. The scenario earthquake is a rupture of a reverse left-lateral oblique fault $2,545.5 \mathrm{~km}$ and $1,800 \mathrm{~km}$ in size in the strike and dip directions, respectively, and the hypocenter is located $12 \mathrm{~km}$ from the surface. The speed of rupture propagation from the hypocenter is $2 \mathrm{~km} / \mathrm{sec}$, and a uniform slip distribution is assumed for the entire fault. The focal mechanism for this scenario is set to be comparable to the focal mechanism of the 2007 Niigata-ken Chuetsu-oki earthquake, ${ }^{9)}$ which affected the operation of a NPP. For the fault-structure model, the epicenter is set $10.18 \mathrm{~km}$ from the NPP; see Fig. 4. The detailed configuration of the NPP is set based on conventional design, ${ }^{10)}$ e.g., the wall thickness is set at $2.0 \mathrm{~m}$, and the base-mat foundation thickness is set at $7.0 \mathrm{~m}$. The modeled NPP is embedded $36.0 \mathrm{~m}$ below the ground surface level on a soft soil layer $80.0 \mathrm{~m}$ deep. This fault-structure system simulation can target ground motion up to $1.0 \mathrm{~Hz}$ and the seismic response of the NPP structure.

We use the MMA with the hybrid-grid FEM to solve the seismic response of the NPP in the above scenario. Following the MMA method, we first conduct the macro analysis and then conducted the micro analysis. For the macro analysis, we set the domain size to include both the fault plane and the NPP; see Table 5. Figure 5 shows the macro model and the position of the epicenter. A realistic irregular surface topography and crust layer interface are incorporated into the simulation model. The entire model is meshed using first-order tetrahedral and tri-linear hexahedral (cube) finite elements. Layer properties are given in Table 6. For each layer, 10 elements are used for one shear wavelength for discretization. This results in a maximum element size of $150.0 \mathrm{~m}$ for the top layer and $300.0 \mathrm{~m}$ for the bottom layer. The fault is modeled using 16 double-point sources. The rupture starting time for each point source is governed by the rupture speed from the hypocenter. Table 7 gives the details of the seismic source. For simplicity, we set the moment magnitude, source time function, and rise time of each point source as identical. Figure 6 shows the settings for the fault plane. Next, we conduct the micro analysis using the results of the macro analysis. Following ${ }^{3)}$, we set the dimensions of the micro analysis model; see Table 5. Figure 7 shows the micro model with the NPP at the center as well as a close-up view. Similar to the macro model, the micro model is meshed using first-order tetrahedral and tri-linear hexahedral (cube) finite elements, and 10 elements are used for one shear wavelength. The maximum element sizes are $100.0 \mathrm{~m}$ and $2.0 \mathrm{~m}$ for the soft soil and NPP, respectively. For the time integration of the macro and micro analyses, the time step is 0.01 seconds for 4096 steps. The final results are bandpass-filtered between $0.1 \mathrm{~Hz}$ and $1.0 \mathrm{~Hz}$.

Figure 8 shows the macro analysis results for the wave- field, influenced by the rupture of the fault and path effects. This (macro) simulation is accurate in obtaining the correct boundary condition for the micro analysis. Figure 9 shows the complex responses of the NPP structure as it is affected by spatial and temporal variations in ground motion. Because this model can be considered a fault-structure system, complicated seismic structural responses can be estimated comprehensively. Figure 10 shows the Fourier amplitude distributions of the macro and micro analysis results for the $\mathrm{x}$ component of displacement around the NPP structure. The difference indicates the correction added to the macro analysis result when local heterogeneities are incorporated into the micro analysis model. Through this correction, a higherresolution solution can be estimated with lower computation cost.

\section{Conclusion}

This paper presents a seismic response analysis of a NPP structure that incorporates a fault-structure system. This procedure, which is based on MMA, efficiently obtains accurate solutions with less computational effort than a direct analysis. Numerical implementation in coding is continuously being improved to ensure that the system can handle realistic conditions that arise in actual problems in both the seismic wave propagation and NPP structural dynamic response. The system can currently only handle low-frequency ground motion $(\mathrm{f} \leq 1.0 \mathrm{~Hz})$ components, but we are in the process of extending the model to include high-frequency components. To account for nonlinear behavior including the failure process, a newly-developed FEM (the Particle Discretization Scheme Finite Element Method) will be incorporated into the system in the near future. ${ }^{11)}$ This proposed method should provide important contributions to seismic analysis and the design of NPP structures as well as significant cost savings.

\section{Acknowledgment}

We thank Dr. Yoshiaki Hisada for his Green's function code used to verify the accuracy of the MMA. The author also acknowledges the support of the Center for Computational Science and e-Systems, Japan Atomic Energy Agency (CCSE-JAEA) and Core Research for Evolutional Science and Technology (CREST) project under Japan Science and Technology Agency. We also gratefully acknowledge the anonymous reviewers whose reviews helped us improve the manuscript. 
Table 1 Domain size for numerical verification model

\begin{tabular}{cc}
\hline & $-32.0 \mathrm{~km} \leq \mathrm{x} \leq 32.0 \mathrm{~km}$ \\
Macro model & $-32.0 \mathrm{~km} \leq \mathrm{y} \leq 32.0 \mathrm{~km}$ \\
& $-60.0 \mathrm{~km} \leq \mathrm{z} \leq 0 \mathrm{~km}$ \\
\hline \multirow{3}{*}{ Micro model } & $-2.5 \mathrm{~km} \leq \mathrm{x} \leq 2.5 \mathrm{~km}$ \\
& $-2.5 \mathrm{~km} \leq \mathrm{y} \leq 2.5 \mathrm{~km}$ \\
& $-5.0 \mathrm{~km} \leq \mathrm{z} \leq 0 \mathrm{~km}$ \\
\hline
\end{tabular}

Table 5 Domain size for application example model

\begin{tabular}{cc}
\hline \multirow{3}{*}{ Macro model } & $-9.6 \mathrm{~km} \leq \mathrm{x} \leq 9.6 \mathrm{~km}$ \\
& $-9.6 \mathrm{~km} \leq \mathrm{y} \leq 9.6 \mathrm{~km}$ \\
& $-15.0 \mathrm{~km} \leq \mathrm{z} \leq$ varies \\
\hline \multirow{3}{*}{ Micro model } & $3.3 \mathrm{~km} \leq \mathrm{x} \leq 6.3 \mathrm{~km}$ \\
& $-6.3 \mathrm{~km} \leq \mathrm{y} \leq-3.3 \mathrm{~km}$ \\
& $-1.5 \mathrm{~km} \leq \mathrm{z} \leq 0.075 \mathrm{~km}$ \\
\hline
\end{tabular}

Table 2 Material properties for numerical verification model

\begin{tabular}{lcc}
\hline & $1^{\text {st }}$ Layer & $2^{\text {nd }}$ Layer \\
\hline Density, $\rho \mathrm{kg} / \mathrm{m}^{3}$ & 2500.0 & 3000.0 \\
Pressure wave velocity, Vp m/s & 4250.0 & 7800.0 \\
Shear wave velocity, Vs m/s & 2500.0 & 5000.0 \\
Quality Factor, Q & 40 & 69.3 \\
\hline
\end{tabular}

Table 3 Point source setting for numerical verification model

\begin{tabular}{lc}
\hline Hypocenter $(\mathrm{x}, \mathrm{y}, \mathrm{z})$ & $(800.0 \mathrm{~m},-600.0 \mathrm{~m},-8500.0 \mathrm{~m})$ \\
& $\mathrm{M}_{o}\left(2 \mathrm{t}^{2} / \mathrm{T}_{o}^{2}\right) \quad 0 \leq \mathrm{t} \leq \mathrm{T}_{o} / 2$ \\
Source function: & $\mathrm{M}_{o}\left(1-2\left(\mathrm{t}-\mathrm{T}_{o}\right)^{2} / \mathrm{T}_{o}^{2}\right) \mathrm{T}_{o} / 2 \leq \mathrm{t} \leq \mathrm{T}_{o}$ \\
& $\mathrm{M}_{o} \mathrm{t} \geq \mathrm{T}_{o}$ \\
Magnitude, $\mathrm{M}_{o}:$ & $1.0 \times 10^{15} \mathrm{~N}-\mathrm{m}$ \\
Strike, Dip, Rake: & $30^{o}, 40^{o}, 60^{o}$ \\
Rise Time, $\mathrm{T}_{o}:$ & $2.0 \mathrm{sec}$ \\
\hline
\end{tabular}

Table 4 Quantitative comparison between MMA and Green's function solution using misfit criteria ${ }^{7,8)}$

\begin{tabular}{cccc}
\hline station & x-comp & y-comp & z-comp \\
\hline R1 & 0.0136 & 0.0129 & 0.0438 \\
R2 & 0.0144 & 0.0124 & 0.0401 \\
R3 & 0.0139 & 0.0139 & 0.0362 \\
R4 & 0.0172 & 0.0160 & 0.0330 \\
R5 & 0.0267 & 0.0207 & 0.0306 \\
R6 & 0.0273 & 0.0218 & 0.0281 \\
\hline \multicolumn{4}{c}{ (a) envelope misfit } \\
\hline station & x-comp & y-comp & z-comp \\
\hline R1 & 0.0041 & 0.0020 & 0.0088 \\
R2 & 0.0040 & 0.0019 & 0.0073 \\
R3 & 0.0050 & 0.0020 & 0.0060 \\
R4 & 0.0056 & 0.0021 & 0.0048 \\
R5 & 0.0054 & 0.0022 & 0.0038 \\
R6 & 0.0041 & 0.0023 & 0.0030 \\
\hline
\end{tabular}

(b) phase misfit
Table 6 Material properties for application example model

\begin{tabular}{lcc}
\hline & \multicolumn{2}{c}{ Macro model } \\
\hline & $1^{\text {st }}$ Layer & $2^{\text {nd }}$ Layer \\
\hline Density, $\rho \mathrm{kg} / \mathrm{m}^{3}$ & 1900.0 & 2500.0 \\
Pressure wave velocity, Vp m/sec & 2300.0 & 4500.0 \\
Shear wave velocity, Vs m/sec & 1500.0 & 3000.0 \\
Quality Factor, Q & 100 & 300 \\
\hline & \multicolumn{3}{l}{ Micro model } \\
\hline & Soft soil & Structure \\
\hline & 1600.0 & 2500.0 \\
\hline Density, $\rho \mathrm{kg} / \mathrm{m}^{3}$ & 1500.0 & 3373.0 \\
Pressure wave velocity, Vp m/sec & 1000.0 & 2127.0 \\
Shear wave velocity, Vs m/sec & 50 & \\
Quality Factor, Q & 5
\end{tabular}

Table 7 Fault parameters of application example

\begin{tabular}{|c|c|}
\hline Hypocenter (x, y, z) & $(-2400.0 \mathrm{~m}, 2400.0 \mathrm{~m},-12000.0 \mathrm{~m})$ \\
\hline & $\mathrm{M}_{o}\left(2 \mathrm{t}^{2} / \mathrm{T}_{o}^{2}\right) \quad 0 \leq \mathrm{t} \leq \mathrm{T}_{o} / 2$ \\
\hline Source function: & $\begin{array}{c}\mathrm{M}_{o}\left(1-2\left(\mathrm{t}-\mathrm{T}_{o}\right)^{2} / \mathrm{T}_{o}^{2}\right) \quad \mathrm{T}_{o} / 2 \leq \mathrm{t} \leq \mathrm{T}_{o} \\
\mathrm{M}_{o} \quad \mathrm{t} \geq \mathrm{T}_{o}\end{array}$ \\
\hline $\begin{array}{l}\text { Moment Magnitude, } \\
\mathbf{M}_{w} \text { : }\end{array}$ & 6.3 \\
\hline Strike, Dip, Rake: & $30^{\circ}, 45^{\circ}, 60^{\circ}$ \\
\hline Rise Time, $\mathrm{T}_{o}$ : & $1.0 \mathrm{sec}$ \\
\hline
\end{tabular}




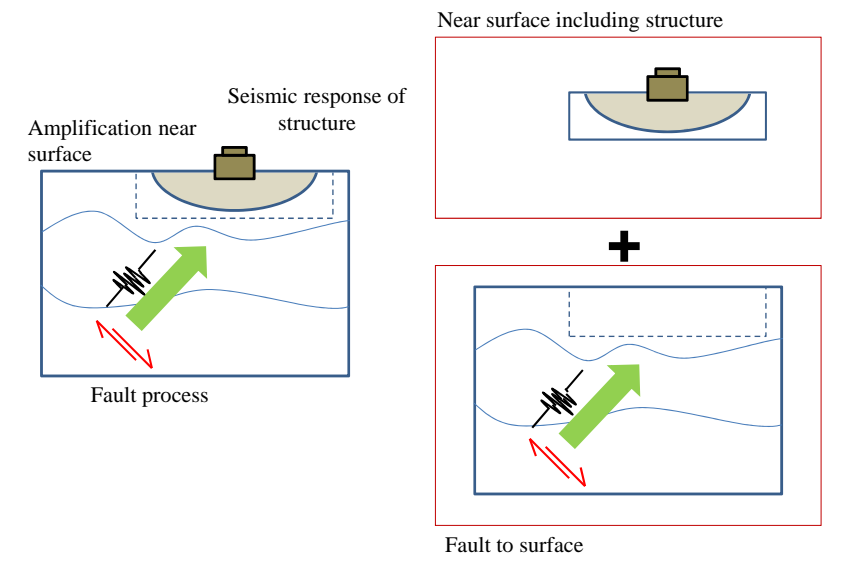

(a)

(b)

Fig. 1 Schematic view of target problem: (a) fault-structure system and (b) decomposed system in MMA. ${ }^{3)}$

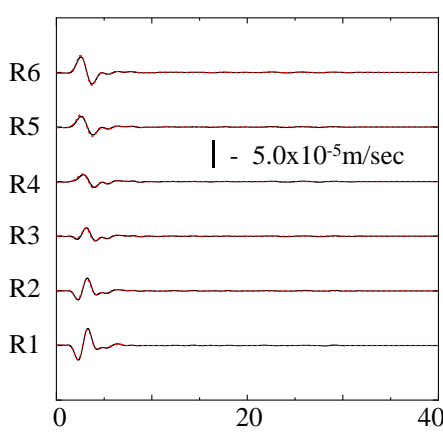

( a )

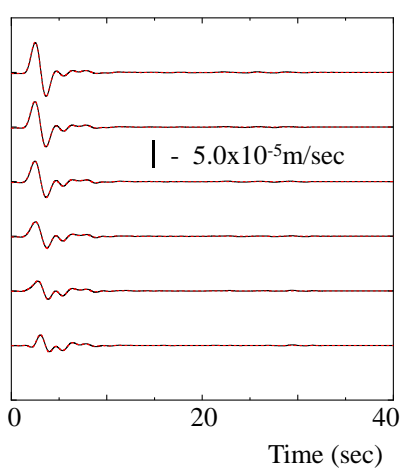

(b)

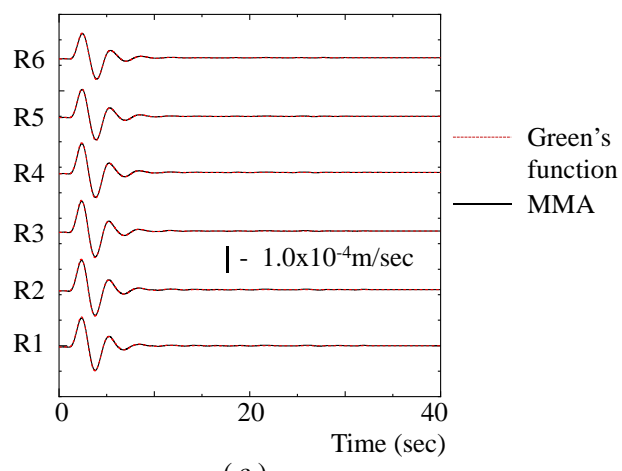

(c)

Fig. 3 Comparison of MMA and Green's function solution at the six receivers, R1 to $\mathrm{R} 6:^{\left.{ }^{7}\right)}$ (a) x-component; (b) ycomponent; (c) z-component.

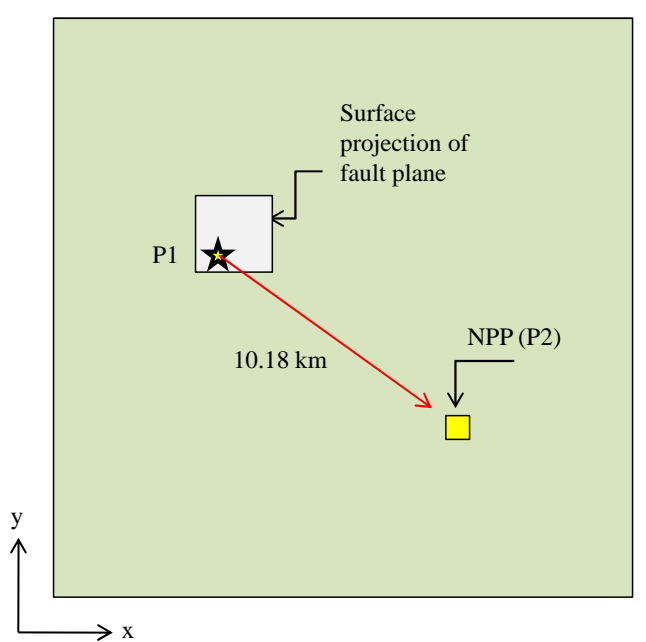

Fig. 4 Target fault-structure system scenario: epicenter, P1 is $10.18 \mathrm{~km}$ from NPP structure, P2.

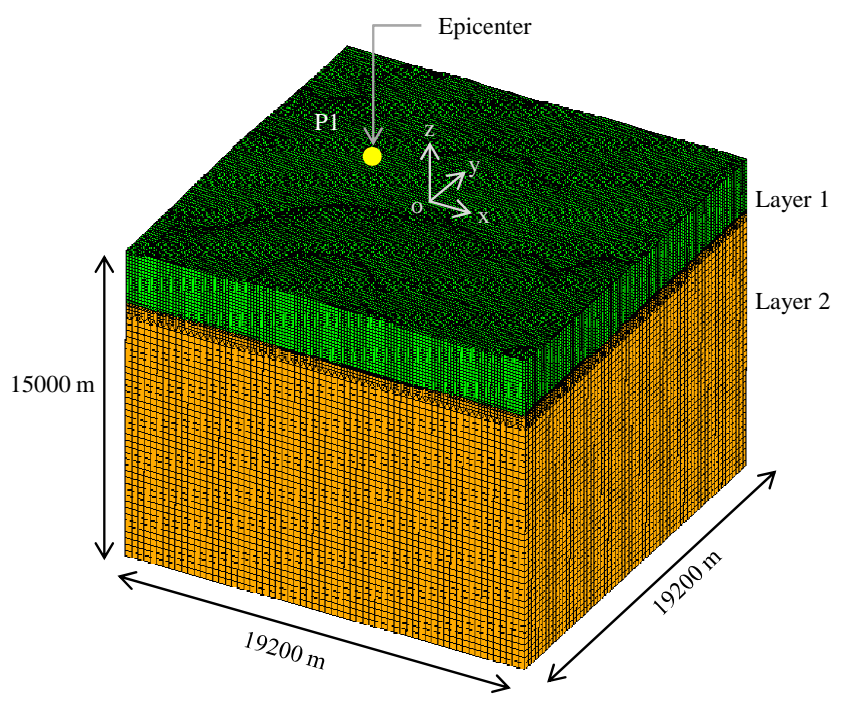

Fig. 5 Macro model which includes irregular topography and layer interface. The model is meshed using 1st-order tetrahedral and tri-linear hexahedral (cube) finite elements. 


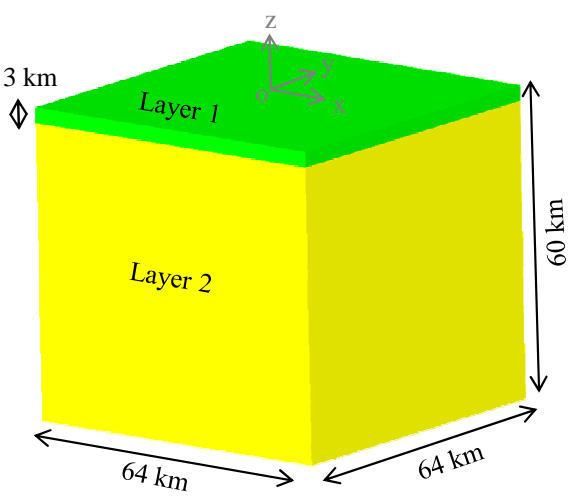

(a )

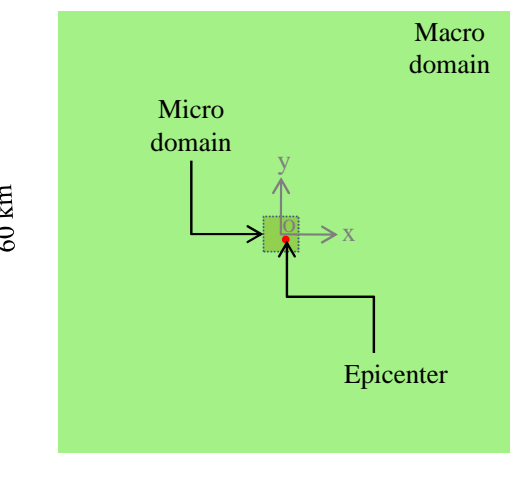

(b)

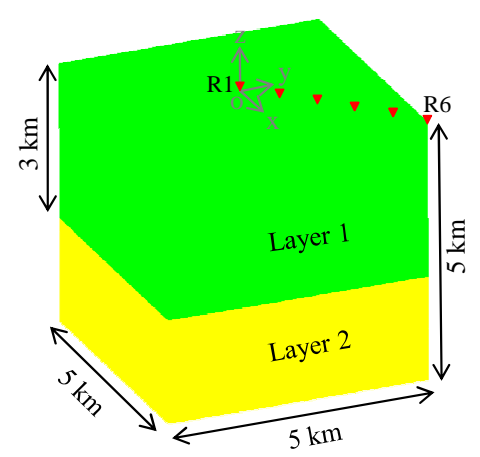

(c)

Fig. 2 Three-dimensional models used in numerical verification: (a) Macro model details; (b) location of Micro model; (c) Micro model details and receivers, R1 to R6.

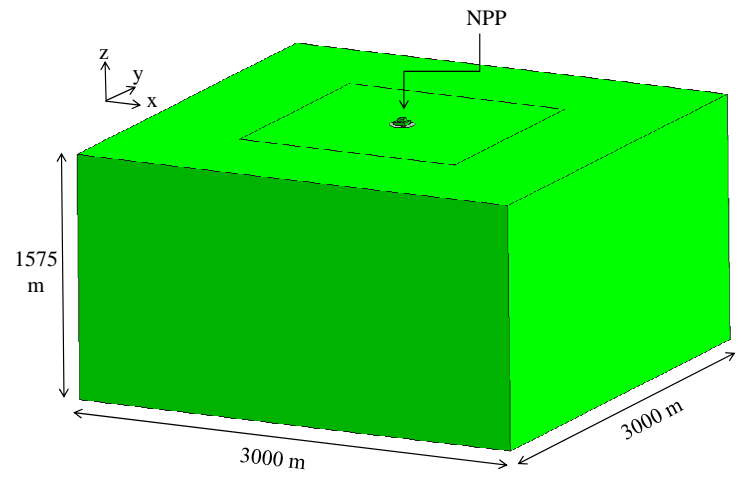

(a)

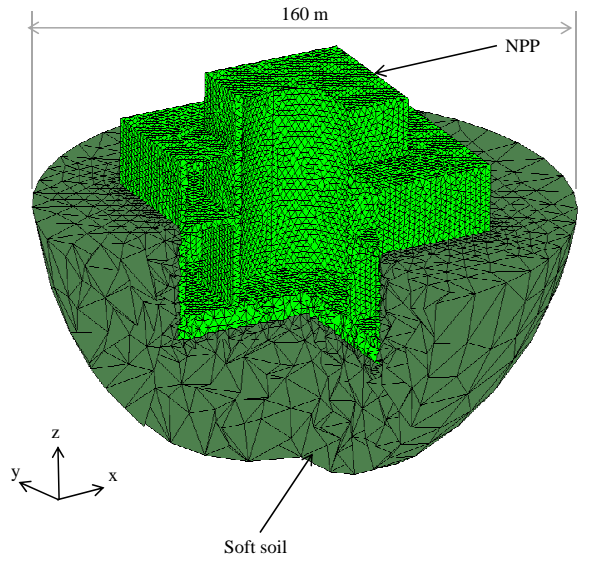

(b)

Fig. 7 Configuration of Micro model: (a) whole-view; (b) close-up view of NPP structure. NPP structure is at the center of this model. NPP structure embedded $36.0 \mathrm{~m}$ in soft soil. 


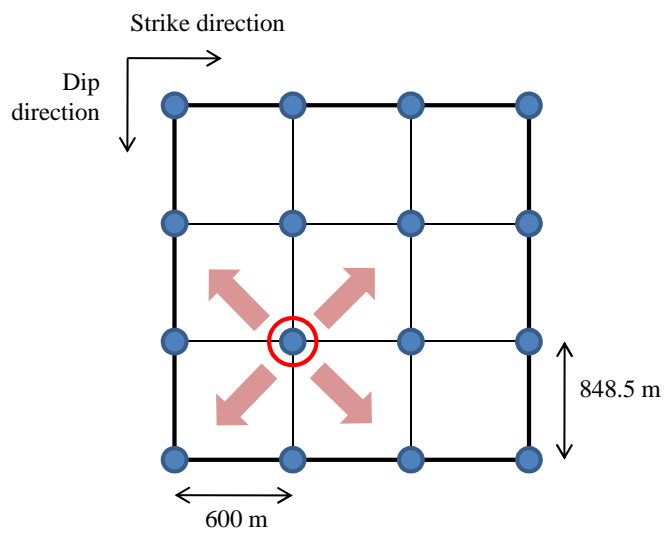

Fig. 6 Fault plane settings. Fault plane is modeled as multiple double-couple point sources (in blue dots) with varying rupture starting time. Hypocenter is in red circle located at coordinates: $(\mathrm{x}=-2400.0, \mathrm{y}=2400.0, \mathrm{z}=-12000.0)$. Rupture speed is $2.0 \mathrm{~km} / \mathrm{sec}$.

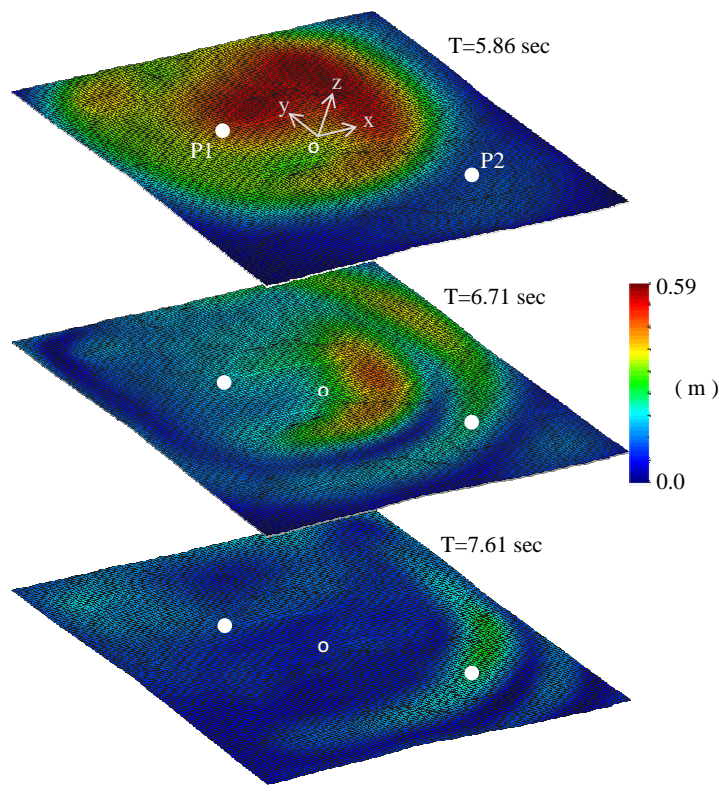

Fig. 8 Snapshots of displacement distribution at surface of macro analysis results. Because of non-uniform starting rupture time of each point source in simple fault-plane and with effect of the nature of crust structure (irregular layer interface and topography) resulting rupture propagation is complicated and exhibits directivity.

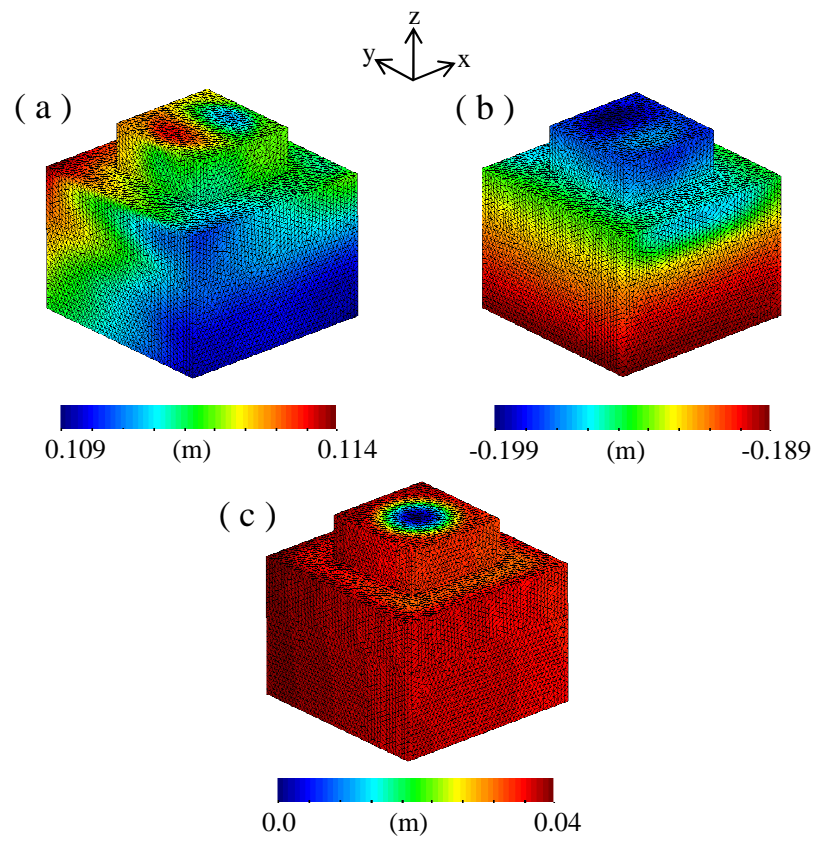

Fig. 9 Seismic displacement response of NPP model at 6.71 second: (a) x-component; (b) y-component; (c) zcomponent. Effect of embedment is evident on the nonuniform lateral response of the structure, (a) and (b). While for the vertical response, the NPP is shown to behave symmetrically with respect to the $\mathrm{z}$-component.

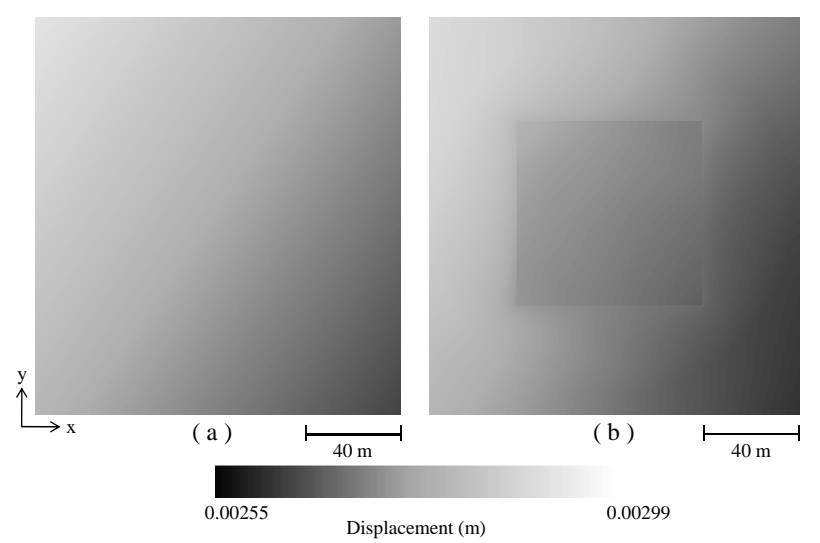

Fig. 10 Distributions of Fourier amplitude component at 0.5 $\mathrm{Hz}$ around NPP structure. (a) and (b) show the result of Macro and Micro analysis, respectively. The difference is the correction added to the Macro analysis result by Micro analysis. 


\section{References}

1) P. Moczo, E. Bystrický, J. Kristek, J. M. Carcione, M. Bouchon, "Hybrid Modeling of P-SV Seismic Motion at Inhomogeneous Viscoelastic Topographic Structures," Bull. Seismol. Soc. Am., 87[5], 1305-1323 (1997).

2) J. Bielak, K. Loukakis, Y. Hisada, C. Yoshimura, "Domain Reduction Method for Three-Dimensional Earthquake Modeling in Localized Regions, Part I: Theory," Bull. Seismol. Soc. Am., 93[2], 817-824 (2003).

3) T. Ichimura, M. Hori, "Structural Seismic Response Analysis based on Multiscale Approach of Computing Fault-Structure System," Earthquake Eng. Struct. Dynam., 38, 439-455 (2009).

4) T. Ichimura, M. Hori, J. Bielak, "A Hybrid Multiresolution Meshing Technique for Finite Element Three-dimensional Earthquake Ground Motion Modeling in Basins Including Topography," Geophys. J. Int., 177, 1221-1232 (2009).

5) J. Bielak, O. Ghattas, E. J. Kim, "Parallel Octree-based Finite Element Method for Large-Scale Earthquake Ground Motion Simulation," Comput. Modell. Eng. Sci., 10, 99-112 (2005).

6) K. Koketsu, H. Fujiwara, Y. Ikegami, "Finite Element Simulation of Seismic Ground Motion with a Voxel Mesh," Pure Appl. Geophys., 161, 2183-2198 (2004).

7) Y. Hisada, "An Efficient Method for Computing Green's Func- tions for a Layered Half-space with Sources and Receivers at Close Depths," Bull. Seismol. Soc. Am., 84, 1456-1472 (1994).

8) M. Kristeková, J. Kristek, P. Moczo, S. Day, "Misfit Criteria for Quantitative Comparison of Seismograms," Bull. Seismol. Soc. Am., 96[5], 1836-1850 (2006).

9) T. Tobita, M. R. Ghayamghamian, G-C. Kang, S. Iai, Preliminary report of the July 16, 2007 Niigata prefecture Chuetsu Off shore (Niigata-ken Chuetsu-Oki), Japan, Earthquake, Disaster Prevention Research Institute, Kyoto University, Japan (2007).

10) H. Abe, Outline of Japanese Seismic Design Review Guide of Nuclear Power Reactor Facilities, Japan Nuclear Energy Safety Organization (2007).

11) M. Hori, K. Oguni, H. Sakaguchi, "Proposal of FEM implemented with particle discretization for analysis of failure phenomena," J. Mech. Phys. Solids, 53, 681-703 (2005).

12) T. Ichimura, M. Hori, H. Kuwamoto, "Earthquake Motion Simulation with Multiscale Finite-Element Analysis on Hybrid Grid," Bull. Seismol. Soc. Am., 97[4], 1133-1143 (2007).

13) Y. Ikegami, K. Koketsu, T. Kimura, H. Miyake, "Finite Element Simulations of Long-period Ground Motions: Japanese Subduction Zone Earthquakes and the 1906 San Francisco Earthquake," J. Seismol., 12, 161-172 (2008). 